\title{
Objective Assessment of Human Workload in Physical Human-robot Cooperation Using Brain Monitoring
}

\author{
AMIRHOSSEIN H. MEMAR, Facebook Reality Labs \\ EHSAN T. ESFAHANI, University at Buffalo
}

\begin{abstract}
The notions of safe and compliant interaction are not sufficient to ensure effective physical human-robot cooperation. To obtain an optimal compliant behavior (e.g., variable impedance/admittance control), assessment techniques are required to measure the effectiveness of the interaction in terms of perceived workload by users. This study investigates electroencephalography (EEG) monitoring as an objective measure to classify workload in cooperative manipulation with compliance. An experimental study is conducted including two types of manipulation (gross and fine) with two admittance levels (low- and high-damping). Performance and self-reported measures indicate that a proper admittance level that enhances perceived workload is taskdependent. This information is used to form a binary classification problem (low- and high-workload) with spectral power density and coherence as the features extracted from EEG data. Using a subject-independent feature selection approach, a subject-dependent Linear Discriminant Analysis (LDA) is used for classification. An average classification rate of $81 \%$ is achieved that indicates the reliability of the proposed approach for assessing human workload in interaction with varying compliance across the gross and fine manipulation. Furthermore, to validate our proposed objective measure of workload, we have conducted a second experiment composed of both fine and gross motor tasks. Compared to interaction with a constant admittance, a lower EEG-based workload is observed with an open-loop variable admittance controller. This observation is in agreement with the subjective workload score (NASA-TLX).
\end{abstract}

CCS Concepts: • Human-centered computing $\rightarrow$ HCI design and evaluation methods; Empirical studies in HCI; • Computer systems organization $\rightarrow$ Robotics;

Additional Key Words and Phrases: Physical human-robot co-operation, electroencephalogram (EEG), workload classification, admittance control

ACM Reference format:

Amirhossein H. Memar and Ehsan T. Esfahani. 2019. Objective Assessment of Human Workload in Physical Human-robot Cooperation Using Brain Monitoring. ACM Trans. Hum.-Robot Interact. 9, 2, Article 13 (December 2019), 21 pages.

https://oi.org/10.1145/3368854

\section{INTRODUCTION}

Physical human-robot cooperation is a rapidly growing field that focuses on the integration of the implicit knowledge of the human operator with the repeatability and accuracy of robots.

This material is based upon work supported by the National Science Foundation under Grant No.1502287.

Authors' addresses: A. H. Memar, Facebook Reality Labs, Redmond, WA 98052; email: amirmemar@fb.com; E. T. Esfahani, University at Buffalo, 200 Lee Road, Buffalo, NY 14228; email: ehsanesf@buffalo.edu.

Permission to make digital or hard copies of all or part of this work for personal or classroom use is granted without fee provided that copies are not made or distributed for profit or commercial advantage and that copies bear this notice and the full citation on the first page. Copyrights for components of this work owned by others than the author(s) must be honored. Abstracting with credit is permitted. To copy otherwise, or republish, to post on servers or to redistribute to lists, requires prior specific permission and/or a fee. Request permissions from permissions@acm.org.

(C) 2019 Copyright held by the owner/author(s). Publication rights licensed to ACM.

2573-9522/2019/12-ART13

https://doi.org/10.1145/3368854 
To obtain a compliant physical interaction, impedance/admittance control methods [Hogan 1984] are widely used in human-robot cooperation. These controllers mimic the behavior of a springmass-damper system in the Cartesian space. In such compliant interactions, it is crucial to properly select the controller parameters (stiffness and damping) to ensure both (i) the stability and (ii) the effectiveness of the interaction, in the sense that it must compromise between physical effort and motion accuracy to minimize the interaction workload perceived by the user [Marayong and Okamura 2004].

It has been shown that a variable controller that modulates its parameters according to both the task requirements and human intention outperforms a constant impedance/admittance scheme. For instance, a low-damping admittance control can facilitate co-manipulation in terms of human physical effort; however, it may degrade the accuracy of fine positioning [Grafakos et al. 2016]. This is mainly due to the fact that humans tend to increase arm stiffness in fine manipulation [Todorov 2004] while the bandwidth of the robot is limited. This makes a low-damping controller over-responsive to input forces. As a result, an intuitive interaction requires lower virtual damping when fast movement without any particular accuracy (gross manipulation) is of interest. However, higher virtual damping for performing fine manipulations is desirable.

The adjustment of the compliance dynamics for achieving a variable admittance/impedance control scheme can be seen as an optimal control problem, where the overall human-robot performance should be optimized. While it is possible to use task-related metrics (e.g., kinematic features of the robot end-effector [Dimeas and Aspragathos 2015; Erden and Marić 2011; Ficuciello et al. 2015; Losey and O'Malley 2018]) in the objective evaluation of pHRI, it is very challenging to model "the perceived workload level" of the human. As a result, the majority of the adaptive admittance controller techniques in pHRI either limit the workload evaluation to subjective measures such as questionnaires or use traditional ergonomic factors such as "physical effort" that focus solely on peripheral outcomes, such as applied force [Duchaine and Gosselin 2007; Lecours et al. 2012; Losey and O'Malley 2018; Mortl et al. 2012] or muscle activity [Grafakos et al. 2016; Song et al. 2013], and disregard the contributions of the brain during physical interaction. Therefore, an objective assessment of perceived workload is required for understanding interaction effects on the users to improve the design of the adaptation strategy accordingly.

This article presents the validity of a brain monitoring approach, based on Electroencephalogram (EEG) recording, as an objective measure to assess physical human-robot cooperations in terms of perceived workload. In particular, we investigate the classification of workload level in cooperative manipulation (co-manipulation) with different dynamics of an admittance controller. Co-manipulation is a common application of admittance control where the robot must freely move under the forces applied by the user. In simple co-manipulations in which the human is the leader, the virtual stiffness is usually set to zero while virtual damping and inertia parameter can be altered. In this regard, estimating the perceived level of workload by subjects via an EEG-based cognitive model can be used either (i) as an offline assessment tool to evaluate the effectiveness of a controller for physical interaction, or (ii) in a real-time manner to maintain the operator workload within the desired range by adjusting the controller parameters. In this article, however, we only focus on case (i).

In our experimental study, the task-load of the co-manipulation is altered by changing the virtual damping in two gross and fine co-manipulation tasks. Statistical analysis for the subjective assessment of the perceived workload reveals the task-dependency of a suitable admittance setup. This is also supported by behavioral assessments based on performance metrics. Our experimental design leads to a task-independent (regarding manipulation type) binary classification problem, since the low- and high-workload conditions involved both the fine and gross co-manipulation tasks. Spectral power density and coherence features are extracted from EEG signals, and a linear 
classifier is used to examine how accurate the EEG features can distinguish between the low- and high-workload condition.

In addition, a secondary experiment is conducted by combining the fine and gross comanipulation to demonstrate how the classifiers trained on the data of the baseline experiment can be used to assess the workload of co-manipulation with a variable admittance controller. Experimental results indicate that EEG classifiers can provide designers with not only the overall workload level of the users upon completion of a task but also useful temporal information regarding the workload variation during the task.

To the authors' knowledge, no existing study has examined the effects of compliant controllers on EEG patterns in cooperation tasks by taking into account both the fine and gross manipulation. The main contribution of this article can be summarized as follows:

(i) This article for the first time investigates the effect of controller dynamics and task type on the brain activity of subjects to identify subject-independent EEG-based physiological features that can predict the perceived level of workload.

(ii) Moreover, the transferability of the EEG-based workload classifier trained on the baseline motor tasks to a more complex motor task is tested. By comparing the results of EEGworkload classifier with the interaction force and subjective workload measures (NASATLX), the article also illustrates the validity of the proposed model.

\section{RELATED WORKS}

The perceived workload can be defined as the portion of operator information processing capacity that is required to meet system demands [van Erp et al. 2010]. Reliable knowledge about the level of workload is a key factor for constructing a human-centered adaptive system. Various methods can be used to assess workload in physical interaction from a qualitative or quantitative point of view. One assessment method is the use of subjective measures via questionnaires. However, these methods are not continuous and cannot be obtained in real-time, since the interruption of the task is required. Moreover, participants' self-reports may be affected by a posteriori rationalization and the desire to satisfy implicit objectives of the researcher [Bethel et al. 2007]. Another approach is the use of performance metrics such as the number of errors, reaction time, or completion time. However, task performance may not be easy to obtain directly when complex motions without a pre-defined path are required.

The current state-of-the-art in pHRI extract information at the peripheral level (e.g., applied force or muscle activity) as an alternative to perceived workload and ignore the contributions of the brain during physical interaction. However, the human mind is a dynamic predictor that perceives, understands, and acts within a dynamic environment. It integrates concurrent multimodal sensory and motor signals to properly execute a task based on its individual's goals. In the case of pHRI, the modification in human behavior can be seen as the modulation of the arm stiffness [Moualeu and Ueda 2015], gripped orientation [Bennis and Roby-Brami 2002], or applied force [Tran et al. 2015]. Therefore, information extracted from interaction force and muscle activity may not resemble the perceived level of workload in physical human-robot interaction.

Recently, neuroergonomic methods such as electrodermal and EEG data analysis have shown promise to assess physical human-robot interactions using real-time quantitative data. For instance, Kulic and Croft [2007] analyzed physiological responses when participants were passively (no physical interaction) observing different motions of a robot manipulator. Participants showed significantly lower skin conductance (less anxiety) with safe motion planners than fast and unsafe robot motions. Dehais et al. [2011] investigated physiological responses of 12 subjects during interaction with a mobile robot in an object hand-over task. Ocular and physiological data including 


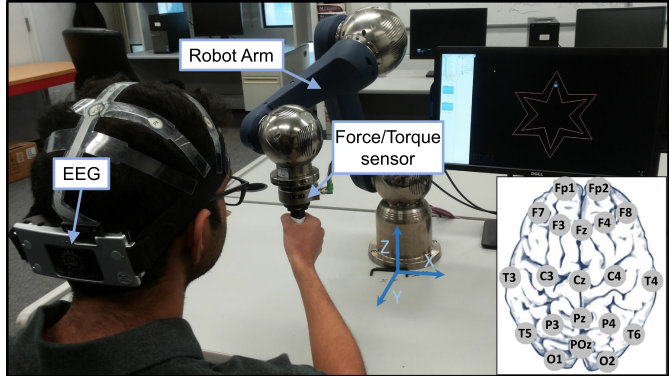

(a)

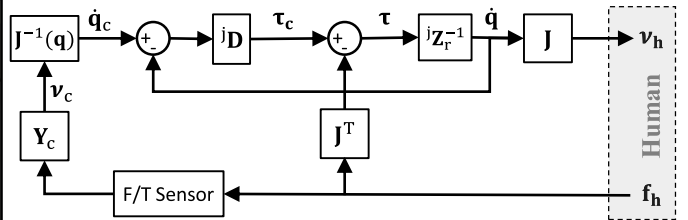

(b)

Fig. 1. (a) Experimental setup and the locations of EEG electrodes on the scalp. (b) The block diagram of the admittance controller.

skin conductance and deltoid muscle activity were used to discriminate between human response to three motion planners with different levels of safety and physical comfort. Novak et al. [2014] used a set of physiological responses to estimate human workload in physical interaction with a gravity- and friction-compensated robot arm. The responses of 10 subjects were recorded while they were asked to play a virtual game with different levels of mental arithmetic and temporal difficulty. All physiological modalities could estimate the workload significantly better than random. Moreover, no significant difference between physiological modalities was revealed regarding accuracy, and combining multiple modalities did not improve accuracy. Although a compliant physical interaction was involved in their study, the effect of robot controller dynamics on the physiological responses and perceived workload were not taken into account.

EEG has become one of the most studied physiological indicators of workload due to its high temporal resolution and recent advances in portable and wearable sensors. However, workload classification from EEG monitoring has been found to be task-specific [Ke et al. 2014]. As a result, satisfactory results are mostly obtained by classifiers trained and tested with EEG features from the same task. This is due to the fact that different cognitive functions need different neural structures and processing strategies that produce different neural activity patterns. In this study, the baseline experiments consisted of two different movements including a simple fine and gross manipulation task. This improves the reliability and generalizability of the workload classifier by selecting EEG features that are common between the two tasks.

\section{MATERIALS AND METHODS}

\subsection{Experimental Setup}

Figure 1(a) illustrates the experimental setup of this study. A 6-DoF lightweight robot arm (SCHUNK PowerBall LWA 4P) was used for co-manipulation and a 6-axis force/torque sensor was attached to its end-effector, since an admittance controller requires the force/torque measurements between the user and robot at the interaction point. A computer monitor was placed in front of the subjects to provide visual information related to the visuomotor tasks and the end-effector position in the X-Y plane.

B-Alert X24 wireless headset, from Advanced Brain Monitoring Inc., was used to acquire noninvasive EEG signals. Sensors were placed at 20 locations based on the 10/20 international EEG system including Fp1, Fp2, F7, F3, Fz, F4, F8, T3, C3, Cz, C4, T4, T5, P3, Pz, P4, T6, O1, POz, and O2 as shown in Figure 1(a). Signals were referenced to linked electrodes located behind each ear on the mastoid bone. 


\subsection{Admittance Control}

An admittance controller was implemented on the robot to provide compliant interaction such that users can lead the robot motions in Cartesian space with null stiffness. Figure 1(b) shows the block diagram of the controller, which consists of two feedback loops: the external feedback loop for controlling the robot admittance and the internal joint-based feedback loop to control the velocity of the joints. In the external feedback loop, a force/torque $(\mathrm{F} / \mathrm{T})$ sensor measures the force between users and robot to compute the desired velocity of the end-effector by taking into account the controller admittance block, $\mathrm{Y}_{c}$, that establishes a first-order relationship between measured force and the desired end-effector velocity as,

$$
\mathrm{Y}_{c}: \quad \mathbf{M}_{d} \dot{v}_{c}+\mathrm{C}_{\mathrm{d}} v_{\mathrm{c}}=\mathrm{f}_{\mathrm{h}},
$$

where $v_{\mathbf{c}} \in \mathbb{R}^{6}$ is the desired Cartesian velocity of the end-effector; $\mathbf{f}_{\mathbf{h}} \in \mathbb{R}^{6}$ is the interaction force and torque vector; $\mathbf{M}_{\mathbf{d}}, \mathrm{C}_{\mathrm{d}} \in \mathbb{R}^{6 \times 6}$ are the admittance gains representing the desired inertia and damping of the system that the controller imposes to the end-effector motions. In our study, the virtual mass $\left(\mathbf{M}_{\mathbf{d}}\right)$ was kept constant, while the virtual damping $\left(\mathbf{C}_{\mathbf{d}}\right)$ was considered as the controller variable to modify the admittance controller. $v_{\mathrm{c}}$ is then transformed into the desired joint space velocities $\left(\dot{\mathbf{q}}_{c}\right)$ based on the inverse Jacobian matrix $\mathbf{J}^{-1}(\mathbf{q})$ by,

$$
\dot{\mathbf{q}}_{c}=\mathbf{J}^{-1}(\mathbf{q}) v_{\mathbf{c}} .
$$

The internal loop attempts to track the desired joint velocities via a closed-loop feedback controller, ${ }^{j} \mathbf{D}$ (motion controller), by exerting torques $\left(\tau_{\mathbf{c}}\right)$ to the robot joints with an intrinsic impedance of ${ }^{j} \mathrm{Z}_{\mathrm{r}}$. A local proportional-integral (PI) controller in each joint can guarantee the tracking of desired joint velocities, since a PI velocity controller is equivalent to a proportionalderivative $(\mathrm{PD})$ position controller.

\subsection{Participants}

18 subjects ( 11 males and 7 females) were recruited via advertisement in the campuses of University at Buffalo to participate in our experimental studies approved by the Institutional Review Board (IRB no. 030-801361). Participants' ages ranged from 23 to 35 years $(M=27.3, S D=3.5)$. None of the participants had experienced human-robot co-manipulation with robot arms and they had normal or corrected to normal vision. Participation in this study was voluntary without compensation.

\subsection{Baseline Experiment}

To investigate the effect of admittance level and task-type on the co-manipulation performance, all the participants were asked to perform four different conditions as the combination of two Manipulation-Type (gross and fine) and two levels of Virtual-Damping (low and high). Figure 2(a) depicts each of the experimental scenarios. Note that only virtual damping was altered in this study, since it has been identified as the most dominant parameter that affects the physical interaction with compliant controllers [Ikeura et al. 1994].

For the gross co-manipulation, participants were asked to guide the end-effector position by turning around two virtual circles displayed on the user's monitor. A spherical cursor was displayed on the monitor as the position feedback of the end-effector. Participants were instructed to generate a path similar to the shape of a rotated " 8 " without particular positioning accuracy or a given path. The primary performance metric of gross co-manipulation was defined as the number of complete turns around the virtual circles in 90 seconds experimental period.

In the case of fine co-manipulation, participants were asked to guide the end-effector precisely between the boundaries of two concentric star-shaped paths (similar to a maze) as illustrated in 


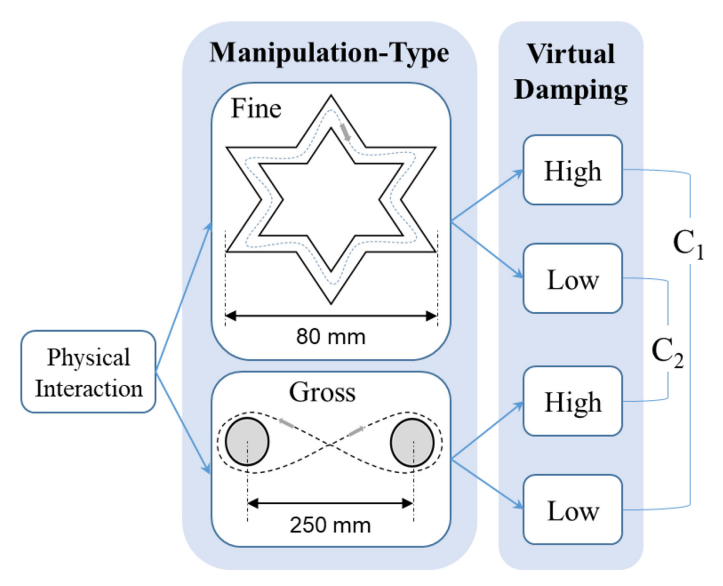

(a)

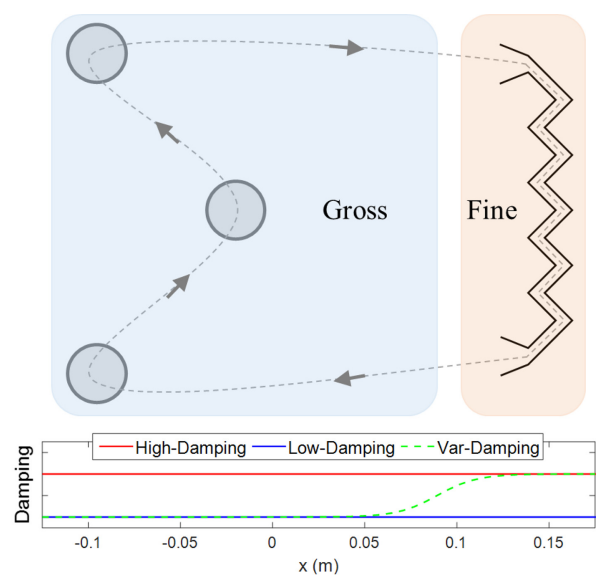

(b)

Fig. 2. (a) Baseline experiment: gross and fine co-manipulation with low- and high-damping setups. Dashed lines represent sample desired paths. All the participants performed this experiment. (b) Secondary experiment: Only 10 participants performed this experiment. The experimental scenario can be divided into gross (left) and fine (right) co-manipulation regions. The transitioning part of green curve defines transition region. The dimensions shown in the figures are measured in the workspace. There is a 1:1.4 ratio between the dimensions in the workspace and the visual display.

Figure 2(a). The primary error metric of the fine co-manipulation was defined as the total number of times that an individual crosses the virtual boundaries within 90 seconds experimental period. Higher intersections represented lower performance in this case.

To compensate the weight of users' arms and increase the interaction safety, no vertical movement of the end-effector was allowed by setting the desired velocity in the Z-direction to zero. For each condition of the experiment, $M_{d}$ and $C_{d}$ were considered as diagonal matrices. The virtual mass of admittance controller was set to $\operatorname{Diag}([3,3,3,0.1,0.1,0.1])$, and the virtual damping was set to $\operatorname{Diag}([80,80,80,10,10,10])$ and $\operatorname{Diag}([20,20,20,6,6,6])$ for the high- and low-damping, respectively.

\subsection{Secondary Experiment}

To further explore the performance of the EEG workload model trained on the data of the baseline experiment, a secondary experiment was designed as a combination of the fine and gross manipulation. Figure 2(b) shows the task scenario for this experiment. The left and right side of the screen corresponded to gross and fine co-manipulation parts, respectively. Ten of the subjects ( 6 males and 4 females-aged $\mathrm{M}=27.8, \mathrm{SD}=3.8$ ) participated in the secondary experiment where they were asked to continuously guide the end-effector position by passing over the virtual circles (gross) and then passing a vertical zig-zag maze while avoiding collisions with boundaries (fine).

Similar to the baseline experiment, no vertical movement of end-effector was allowed. This experiment was performed by the participants with three different admittance setups, including (i) constant low-damping, (ii) constant high-damping, and (iii) variable-damping. Matrix $C_{d}$ for low- and high-damping setups was similar to the baseline experiment. For variable-damping, a hyperbolic tangent function was used to gradually switch between low- and high-damping as a function of the end-effector position in the $\mathrm{X}$ coordinate. Damping altered from the low to high setting for moving from the gross to the fine region and vice versa. 


\subsection{Experimental Procedure}

After briefing participants on the objectives of the experiment, they received training and then practiced on the experimental setup to make sure that they had gained adequate mastery to accomplish the tasks independently. In the training session, the basic concept of robot guidance with admittance control was introduced. Then, the fine and gross co-manipulation tasks and their primary performance metrics were described.

A sample video recorded from an operator performing the two co-manipulation types was used in the training session. Then, participants performed a practice session with the low- and highdamping setup and experienced co-manipulation in free space. A two-minute practice session was offered for each damping condition to familiarize the subjects with the behavior of the admittance controller. Extra practice time was provided, in case a participant requested more practice. Only 2 out of 18 participants requested extra time for practice.

To balance the learning effects of the main experiment, the order of task conditions was permuted randomly for each subject. In the baseline experiment, the order of tasks was permuted, so the first and last two tasks were a same manipulation type. This constraint was applied to ease the subjective comparison between the low- and high-damping for each manipulation type. In the secondary experiment, simple randomization was applied on the damping conditions. Upon the ending of each condition, participants were asked to complete a NASA TLX questionnaire [Hart and Staveland 1988] as a subjective workload assessment. The NASA TLX included six taskload dimensions: Mental Demand, Physical Demand, Temporal Demand, Performance, Effort, and Frustration.

\subsection{EEG Data Processing and Feature Extraction}

EEG signals were recorded with a sampling rate of $256 \mathrm{~Hz}$ and 16 bits/sample resolution. Preprocessing was performed using the EEGLAB toolbox for MATLAB [Delorme and Makeig 2004]. EEG signals were band-pass filtered using a two-way least-squares FIR filter in the range of 0.3 to $50 \mathrm{~Hz}$. Independent Component Analysis (ICA) was used for artifact removal using the extended InfoMax method as implemented in the EEGLAB. Independent components predominantly driven by sources of artifact (eye-blinks, eye-movements, and muscle activity) were visually identified based on IC topography, spectral curve, and time-course activity, and then were removed from the recorded signals. The ICA-pruned continuous data were then segmented into two-second epochs for feature extraction.

Various features have been used in the literature for EEG-based classification of different mental states. Generally EEG features can be distinguished in time domain, frequency domain, and time-frequency domain. A complete review of the EEG feature extraction for affective computing and workload estimations is provided in Jenke et al. [2014]. The amplitude and latency of Event Related Potentials (ERPs) are the most common time domain features, which are not applicable to continuous and single trail EEG recording where there is no time-locked stimuli [Blackwood and Muir 1990]. Other time domain EEG features such as Hjorth parameters [Hjorth 1970] that capture statistical properties of EEG signals, Largest Lyapunov Exponent [Esfahani and Sundararajan 2011], and autoregressive coefficients [Anderson et al. 1998] can be highly subject-dependent and difficult to physiologically interpret.

The most popular features in the context of cognitive state recognition from EEG are related to frequency domain, since oscillations are considered to be the fundamental neural mechanism that enable the synchronization of neural activity within and across brain regions [Jenke et al. 2014]. Band power is the most common frequency domain feature and different algorithms have been proposed to calculate band power, such as fast Fourier transform (FFT), short-time Fourier transform 


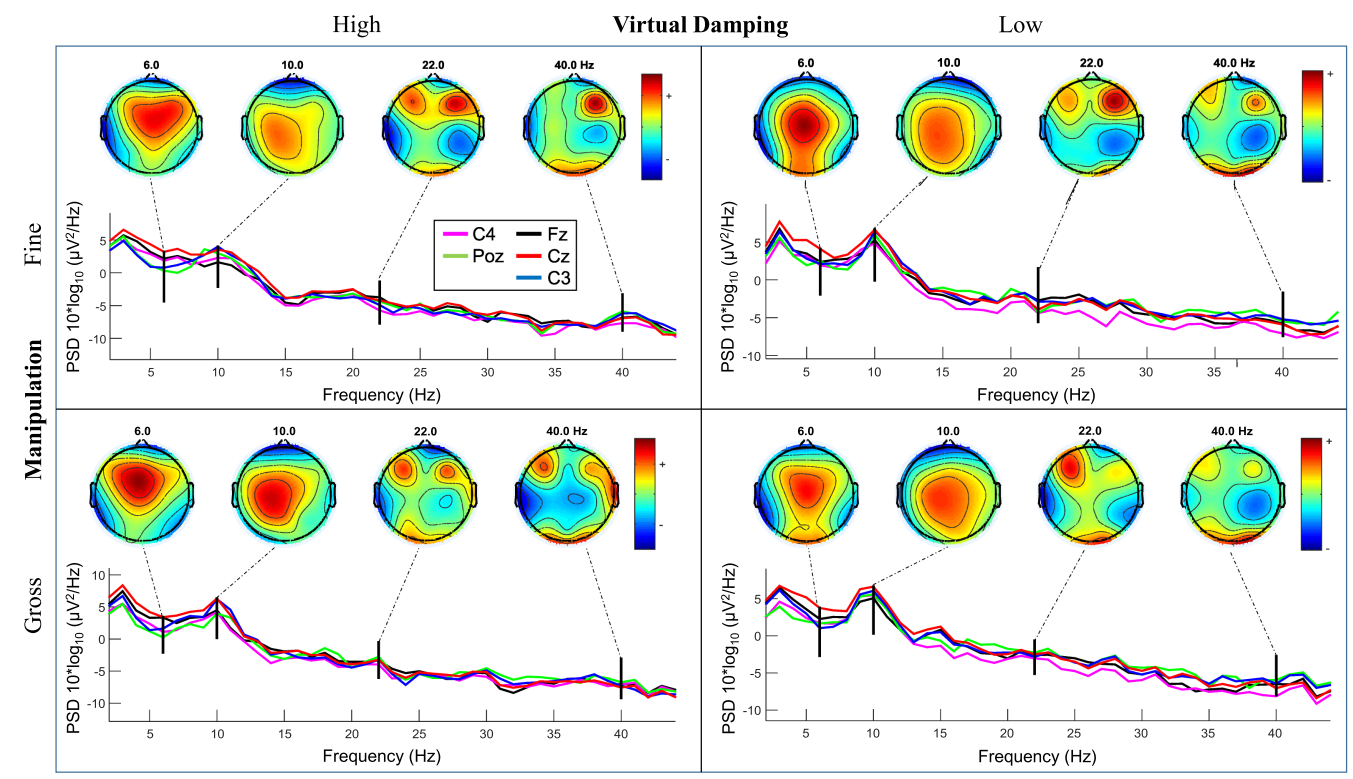

Fig. 3. The mean scalp maps of spectral power density between the experimental conditions for one of the subjects (S3).

(STFT), or the estimation of spectral power density using Welch's method. Time-frequency methods for EEG feature extraction provide the capability of studying EEG patterns in both the time and frequency domains simultaneously. Discrete Wavelet Transform and Hilbert-Huang Spectrum are the most common time-frequency methods in EEG analysis [Cohen 2014]. These methods are suitable tools for analyzing short duration EEG data such as motor imagery [Qin and He 2005], visual imagery [Esfahani and Sundararajan 2012], and ERP analysis [Cohen 2014].

In addition to the EEG features that only capture the activity of a single electrode, several methods have been used in the literature to measure the functional connectivity between electrodes. Linear power-based connectivity (e.g., spectral coherence), mutual information methods, and Granger causality are the most common functional connectivity methods [Cohen 2014]. In this study, spectral power density and coherence features are used due to their robustness over time and low computational cost for real-time analysis.

3.7.1 Spectral Power Density. Power spectral analysis was used for feature extraction in this study, since it is a well-established method (as reviewed in Bashashati et al. [2007] and Jenke et al. [2014]) for the analysis of EEG signals, representing the distribution of the signal power over the frequency bands. From the filtered and decontaminated EEG signals, spectral power densities were extracted from each two-second epoch using Welch's estimator. Each epoch was divided into eight Hamming windowed segments with $50 \%$ overlap to improve the estimation quality. Figure 3 demonstrates the topographic mapping of EEG spectral power and corresponding periodogram curves at five channels (Fz, Cz, C3, C4, POz) for one of the subjects (S3). The obtained periodograms were averaged to estimate spectral power density in $1-\mathrm{Hz}$ bins and then averaged across 6 frequency bands including theta $[4-7 \mathrm{~Hz}]$, lower-alpha [8-10 Hz], higher-alpha [11-13 Hz], lowerbeta [14-22 Hz], higher-beta [23-35 Hz], and gamma [36-44 Hz]. As a result, a feature vector of 120 elements $\left(N_{c h} \times N_{f}\right.$ where $N_{c h}=20$ and $N_{f}=6$ are the number of channels and bands, respectively) were extracted from spectral power analysis for each epoch. 
3.7.2 Functional Connectivity. Spectral Coherence was used to estimate functional connectivity between channel pairs, since it is a well-established method to measure linear dependency between two time series in the frequency domain [Srinivasan et al. 2007]. Spectral coherence quantifies the level of synchrony between two signals at a specific frequency and ranges from 0 to 1 . A high coherence implies extensive communication between the two regions of the brain, whereas a low coherence corresponds to less connectivity indicating higher regional independency.

Welch's method was used to estimate EEG coherence, with the same parameters used to estimate spectral power. Coherence values were also averaged across the six aforementioned frequency bands. All the combination of channel pairs in six bands will lead to a total number of 1140 features for each epoch $\left(\frac{N_{c h} \times\left(N_{c h}-1\right)}{2} \times N_{f}\right)$. However, in this study, only the coherences of EEG channels with $(\mathrm{Fz})$ were taken into account. As a result, a feature vector of 114 elements $\left(\left(N_{c h}-1\right) \times N_{f}\right)$ was obtained from coherence analysis for each two-second epoch.

Functional connectivity between the frontal midline (Fz) and motor, temporal, parietal, and occipital regions are extensively studied in neuropsychology when movement-related tasks are of interest. These connectivities have correlations with motor planning, somatosensory, and visuomotor integration processes [Hatfield et al. 2004]. For instance, experts demonstrate a more efficient cortical function in motor planning, in which non-essential interactions between the motor planning and other regions of the brain are suppressed [Deeny et al. 2009]. Moreover, the spectral coherence of $\mathrm{Fz}$ channel with the other cortical regions has been found as an indicator of motor-task difficulty [Rietschel et al. 2012].

\subsection{Feature Selection and Classification}

A total of 234 features were extracted from each two-second epoch by combining EEG spectral power density and coherence features. To label the extracted epochs of each experimental scenario, a binary classification approach with two classes of low- $\left(C_{1}\right)$ and high-workload condition $\left(C_{2}\right)$ was defined. Low-damping gross manipulation and high-damping fine manipulation were labeled as the low-workload, whereas the two other conditions were labeled as the high-workload class as shown in Figure 2(a). This labeling criterion was supported by the results of performance and subjective assessment that are presented in Sections 4.1 and 4.2. Also, using this labeling will provide us with a balanced binary classification problem where the chance would be $50 \%$.

A Stepwise Linear Discriminant Analysis (SWLDA) was utilized for reducing the dimensionality of the feature space to avoid over-training by selecting features that offer the highest independent discriminative information. SWLDA has been widely used for feature selection in brain-computer interfaces and EEG-based affective state classification [Aricò et al. 2016; Berka et al. 2007]. It is a numerical approach that iteratively adds/removes features with significant/insignificant discrimination effect. Although SWLDA cannot handle nonlinear relationships in the feature space, it is a simple and robust feature selection method for EEG data. In Krusienski et al. [2006], a comparison between five classifiers demonstrated the competitive feature selection and classification performance of SWLDA method for EEG data. The Mahalanobis distance was used for SWLDA to add or remove features, with a threshold of 0.01 on p-value for adding and 0.1 for removing a variable.

Since the model used for the discriminant analysis assumes features form a multivariate normal distribution, a logarithmic transformation was applied to the spectral power [Gasser et al. 1982] and Fisher's Z-transformation on the coherence to normalize their distributions [Amjad et al. 1997].

For the first experiment, a 4-fold cross-validation procedure was used for feature selection and classification purposes. Data of each subject are randomly partitioned into 4 equal size subsamples (tasks distributions are balanced). At each round, 75\% of each subject's data are used for training 


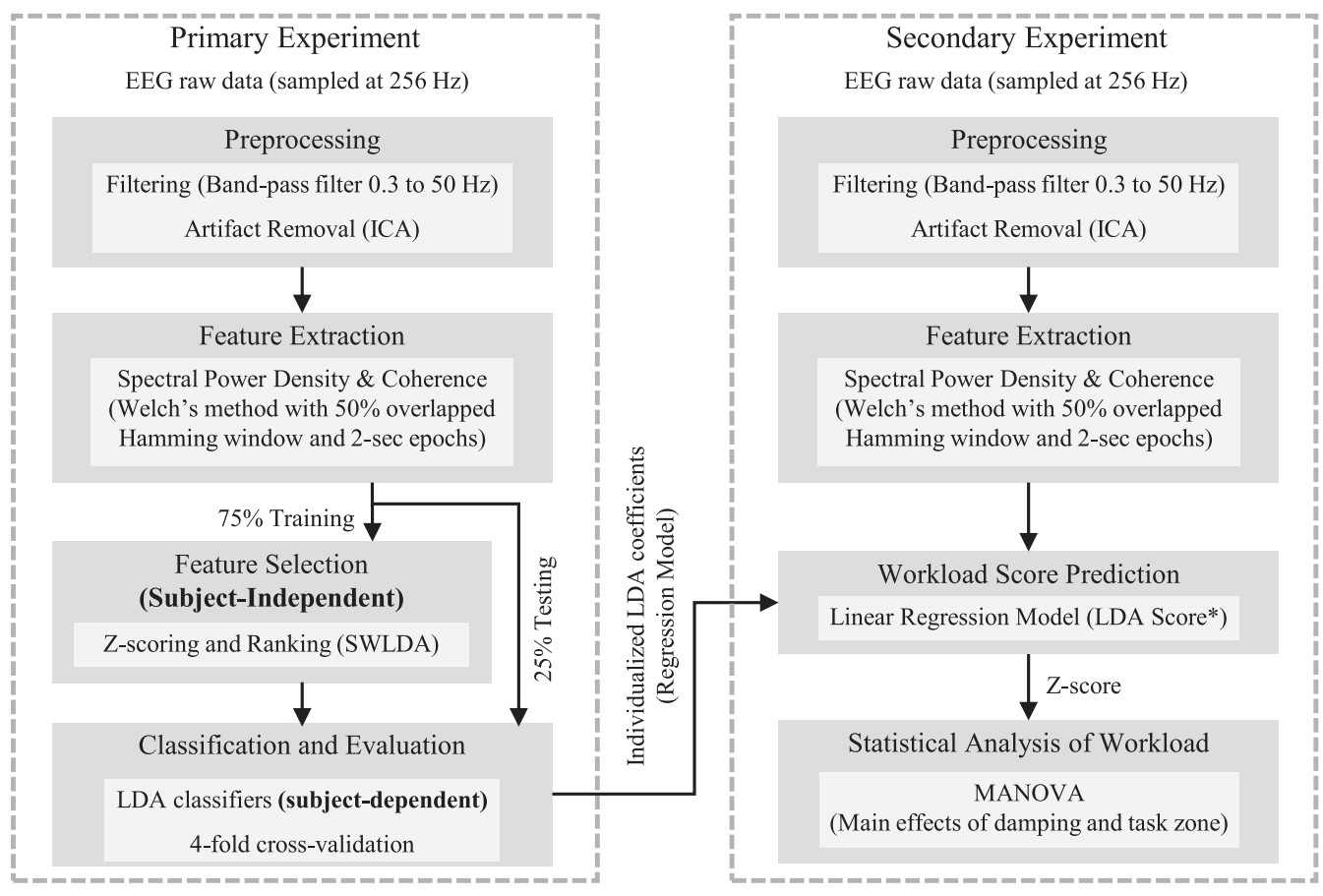

Fig. 4. Flowchart of the EEG data processing pipeline. *Note that in binary LDA, the classification weights are identical to the weights of a linear regression model.

and the remaining for testing. Extracted features from training data of all the participants were merged to find the most dominant subject-independent features that can discriminate between the two classes. As a result, a total of 2,430 samples (135 epochs per subject) were used for this purpose. To remove the influence of individual differences in the feature selection, features were transformed into z-scores based on the distribution of each individual.

Linear Discriminant Analysis (LDA) was utilized for the classification purposes. LDA finds the mapping between the feature and label space through a linear transformation matrix that maximizes a so-called Fisher discriminant ratio [McLachlan 2004]. While the selected features were the same across all subjects, LDA was only trained on each subject's dataset to further remove the effect of individual differences. Finally, to evaluate the transferability of the learned model on primitive tasks to a more complex task with unlabeled data, the individualized linear regression model utilizing the LDA coefficients from the baseline experiment were used to obtain a continuous workload score for the statistical workload analysis of the secondary experiment. For this purpose, the same feature selection and classification approach was used on all data recorded in the first experiment. The overall pipeline for EEG signal processing and using LDA coefficients for the workload prediction of the secondary experiment is shown in the flowchart of Figure 4.

\section{RESULTS}

\subsection{Behavioral Assessment}

Behavioral data were analyzed primarily to examine the validity of the experimental design and the task difficulty manipulation. To acquire insight into the experimental scenarios and provide a visual comparison between them, the trajectories tracked by a single subject are depicted in Figure 5(a). The number of complete turns was considered as the performance of the gross 

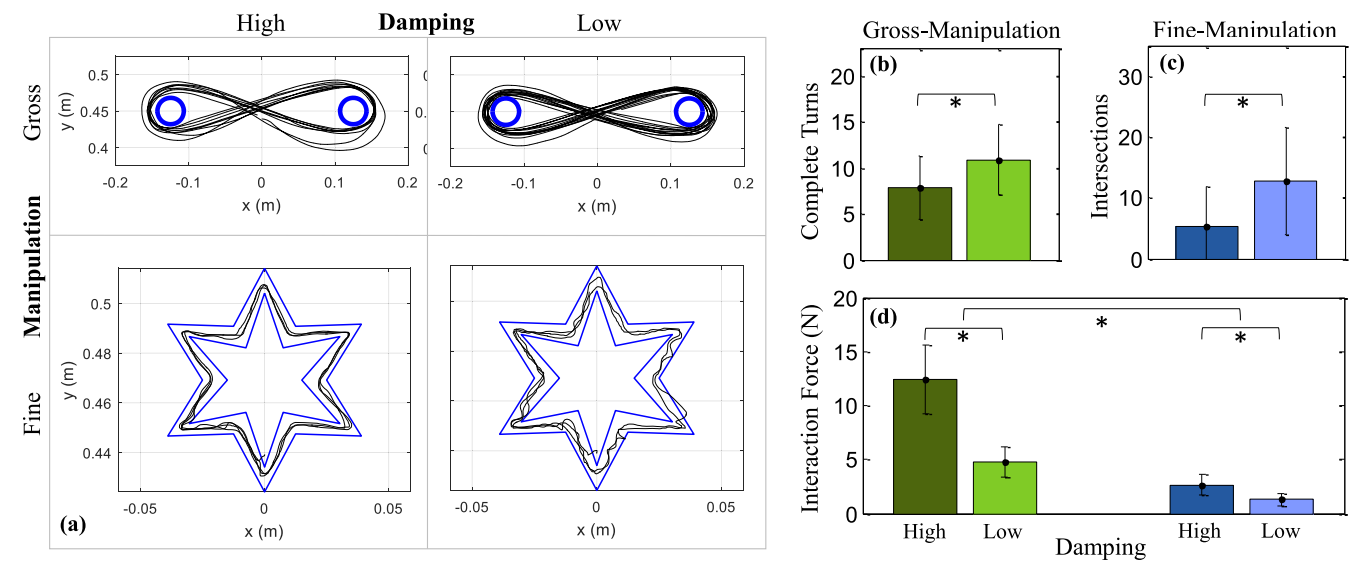

Fig. 5. (a) The tracked paths by one of the subjects in the four experimental conditions. (b) and (c) show the bar plots for the performance metrics of the gross and fine co-manipulation. The darker color of bar plots in each manipulation-type indicates higher damping. (d) represents the average magnitude of interaction forces. Error bars on the graphs represent one standard deviation and * markers denote the significant difference of pairwise comparisons at 0.05 level.

co-manipulation, whereas the number of intersections with boundaries was defined as the error metric of the fine co-manipulation. Two separate metrics were defined to identify the performance of the subjects in each co-manipulation task, since the nature and objective of the tasks were different. Moreover, these metrics were used to examine the effect of damping level on the performance within each task-type rather than between them.

Paired-sample t-tests were performed to compare participants' performance within each comanipulation scenario. Significant differences are reported based on their t-statistic and degreeof-freedom, $t(\mathrm{df})$, as well as the corresponding $\mathrm{p}$-value. The mean performances for each interaction condition are shown in Figures 5(b) and (c). For the gross co-manipulation, the number of complete turns around the virtual circles was considered as the primary performance metric. Statistical analysis revealed a significantly lower number of turns in the high-damping condition than low-damping $(t(17)=-4.7, p<.001)$. In the fine co-manipulation, the number of intersections between tracked trajectory by the users and defined boundaries was used as the primary performance measure. Contrary to the gross manipulation, paired-sample t-test revealed a significantly higher performance with high-damping than the low-damping condition in the fine co-manipulation $(t(17)=-4.16, p<.001)$.

The relatively large variation in the performance metrics across the subjects is mostly due to the individual differences in physical strength and arm impedance characteristics. Subjects with higher strength could apply larger forces and perform faster particularly in the gross co-manipulation while a large arm impedance (especially the arm stiffness) may influence the level of interaction stability in the fine co-manipulation.

In addition to the primary performance measures, admittance/impedance controllers are also evaluated based on their effectiveness in reducing the average interaction force [Ficuciello et al. 2015]. Thus, the average magnitude of interaction forces in the X-Y plane $\left(\frac{1}{N} \sum_{i=1}^{N}\left\|f_{h}\left(t_{i}\right)\right\|\right.$ where $f_{h}$ is the force vector in X-Y plane) was computed to further explore the interaction. The mean forces of the four co-manipulation conditions are presented in Figure 5(d).

A 2 by 2 [Manipulation-Type (gross and fine) $\times$ Virtual-Damping (low and high)] repeated measures analysis of variance (ANOVA) was used for statistical analysis. Significant main effects are 

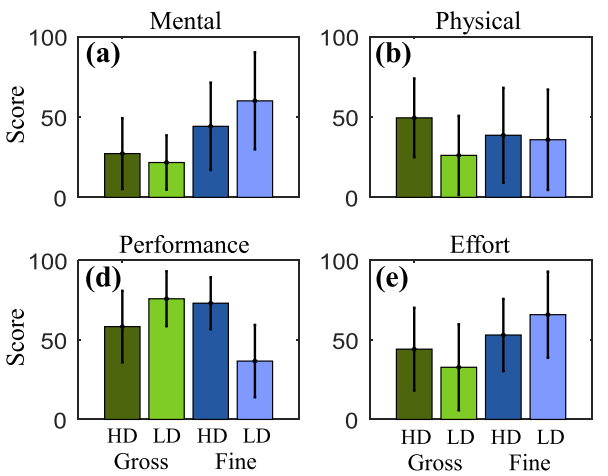
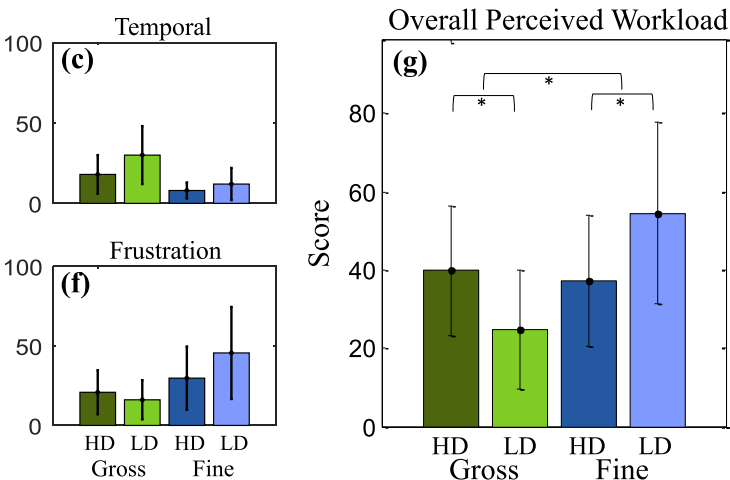

Fig. 6. Results of subjective workload assessment. (a-f) illustrate the six task-load dimensions of NASA-TLX questionnaire. (g) represents the combined weighted perceived workload.

Table 1. Average NASA-TLX Weights Obtained from Paired Comparisons between the Six Workload Dimensions by the Subjects

\begin{tabular}{|c|c|c|c|c|c|c|}
\hline & Mental Demand & Physical Demand & Temporal Demand & Performance & Effort & Frustration \\
\hline 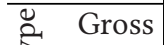 & 0.20 & 0.27 & 0.04 & 0.30 & 0.12 & 0.07 \\
\hline Fine & 0.19 & 0.28 & 0.02 & 0.31 & 0.12 & 0.08 \\
\hline
\end{tabular}

reported based on their F-statistic and degree-of-freedoms, $F$ (hypothesis $\mathrm{df}$, error $\mathrm{df}$ ), as well as the corresponding $\mathrm{p}$-value and effect size $\left(\eta_{p}^{2}\right)$. ANOVA for the average force revealed a main effect for Manipulation-Type $\left(F(1,17)=223.9, p<.001, \eta_{p}^{2}=.93\right)$, indicating that the magnitude of interaction force for gross was higher than fine co-manipulation. A main effect was also revealed for Virtual-Damping $\left(F(1,17)=144.1, p<.001, \eta_{p}^{2}=.89\right)$. Test of simple main effects indicated that by decreasing Virtual-Damping, interaction forces decreased in both gross $(p<.001)$ and fine co-manipulation $(p<.001)$. A significant Manipulation-Type $\times$ Virtual-Damping interaction $\left(F(1,17)=50.5, p<.001, \eta_{p}^{2}=.75\right)$ was also observed.

\subsection{Subjective Assessment}

Figure 6 shows the results of self-reported workload scores ranged from 0 to 100 using NASA TLX questionnaire. The six raw TLX components (Figures 6(a-f)) indicate the relative importance of Performance, Physical, Effort, and Mental workload in the presented co-manipulation tasks, in the sense that their reported scores are relatively higher than Temporal and Frustration components. In addition to the raw scores, a total workload score was calculated using a weighted combination of the six dimensions of NASA TLX for statistical analysis. Weights of the six dimensions were calculated following a set of 15 paired comparisons between the six workload dimensions as described in Hart and Staveland [1988]. The average weights for the gross and fine manipulation tasks are listed in Table 1 and are shown to follow the same pattern. It is not surprising to see the temporal demand and frustration dimensions to have the lowest weights, as the secondary experiment does not impose any temporal constraints or objectives.

Mental and physical demands are both task-related scales, as they focus on the objective demands of the experimental tasks. The effort dimension is a subjective evaluation that represents how hard (both mentally and physically) the participants tried to satisfy the task requirements. The mental or cognitive aspect of the effort is an important factor in motor task [Lee et al. 1994] especially when 


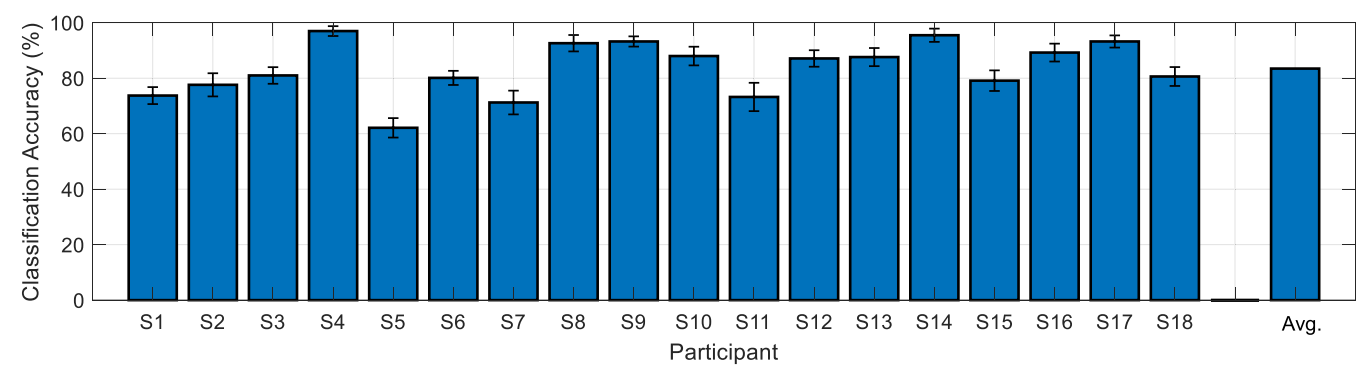

Fig. 7. Classification accuracy for each subject. The last bar plot shows the average accuracy over the participants.

the stability of the movement can be affected [Akizuki and Ohashi 2015]. The physical aspect, like the physical demand dimension, is affected by the damping conditions in the experiment regardless of the type of motor control strategy. In Figures 6(a) and (e), a similar trend in mental demand and effort can be observed.

The performance dimension is a behavior-related scale indicating the perceived quality of the task operated by the participants [Hart and Staveland 1988]. It reflects the perceived gap between participants' desired state and actual state. In this experiment, the desired state is to perform the task with minimum error and effort. This gap is minimized when the damping level is properly adjusted (Gross-LD and Fine-HD as shown in Figure 6(d)). For the other two conditions, the gap is increased and therefore the performance is decreased. In the case of Fine-LD, the movement can be easily unstable, which imposes a much higher level of mental demand (and requires higher effort) as indicated in the Figure 6(a). The case of Gross-HD does not destabilize the movements but simply is more physically demanding as shown in Figure 6(b). A 2 by 2 repeated measures ANOVA revealed a main effect for Manipulation-Type $\left(F(1,17)=31.3, p<.001, \eta_{p}^{2}=.65\right)$, indicating that the perceived workload for the fine was higher than gross co-manipulation. A significant interaction between Manipulation-Type and Virtual-Damping $\left(F(1,17)=55.5, p<.001, \eta_{p}^{2}=.76\right)$ confirmed the task-dependency of suitable damping for co-manipulation tasks using admittance control. Test of simple main effects revealed that by decreasing Virtual-Damping in gross co-manipulation the perceived workload decreased $(p<.001)$, while a completely opposite result was obtained for the fine co-manipulation $(p<.001)$.

\subsection{Classification}

The stepwise linear discriminant analysis yielded a significant result (Wilk's $\lambda=.71, p<.001$ ). Wilk's $\lambda$ indicates the significance of the discriminant functions and ranges from 0 to 1 . The average correct classification rate after 4-fold cross-validation was used to assess the performance of LDA classifier for each individual. The results are shown in the bar plots of Figure 7, and the average classification performance across all the subjects was around $81 \%$. The confusion matrix averaged over all the subjects is also shown in Table 2. The results confirm that classifiers trained on the selected set of EEG features can successfully differentiate between the two low- and highworkload conditions for the fine and gross co-manipulation tasks.

\subsection{Results of the Secondary Experiment}

To further evaluate the workload classifier on a more general co-manipulation task, a secondary experiment including both of the manipulation types was considered. The goal of this experiment was to demonstrate how the information of EEG classifiers trained on the results of the baseline experiment can be used to analyze more complex co-manipulation scenarios. For this purpose, 
Table 2. Average Confusion Matrix over the Subjects

\begin{tabular}{cc|cc}
\hline & & \multicolumn{2}{|c}{ Predicted } \\
& & Low-WL & High-WL \\
\hline$\overline{\widetilde{J}}$ & Low-WL & $81.3 \pm 11.1$ & $18.7 \pm 11.1$ \\
䒧 & High-WL & $18.4 \pm 9.7$ & $81.6 \pm 9.7$ \\
\hline
\end{tabular}
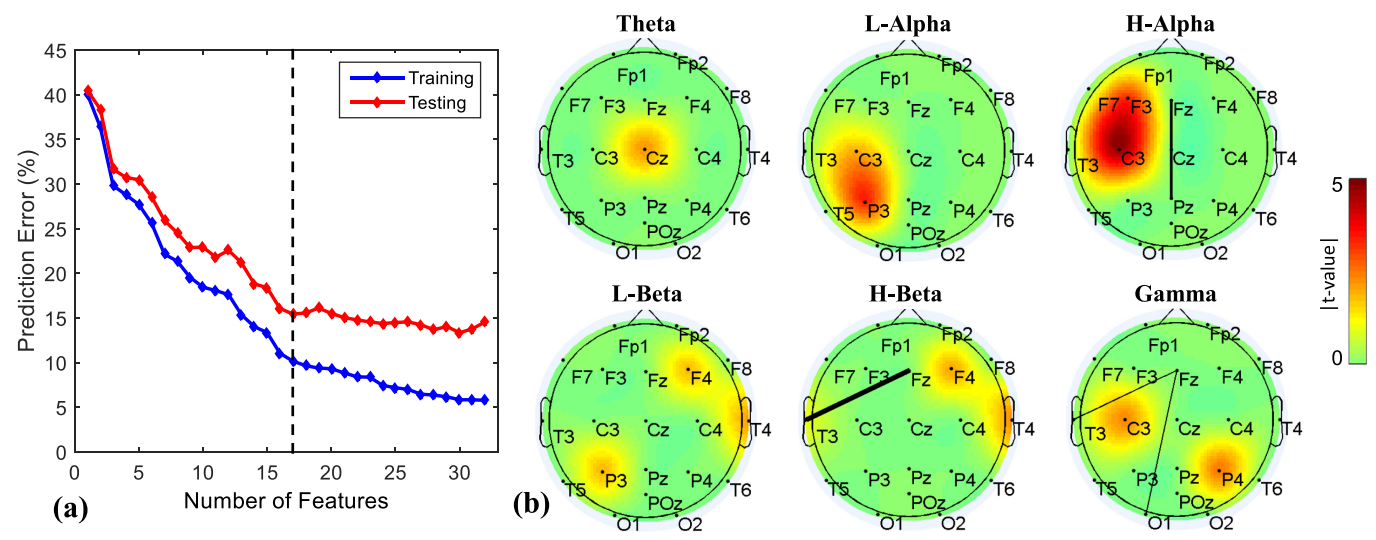

Fig. 8. (a) Train/Test performance plot. The vertical dashed line shows the selected number of features. (b) Final selected set of EEG features. Each topographic map corresponds to a certain frequency band. Obtained t-statistics were used to define the dominance of each feature based on a color map for spectral power and line thickness for coherence.

10 of the recruited subjects (S1 to S10 in Figure 7) performed the experiment shown in Figure 2(b) and their EEG data were recorded for workload analysis with two-second epochs. We repeated the feature selection method over all the data of all the subjects recorded in the first experiment and repeated the feature selection process. The primary feature set obtained from SWLDA included 33 EEG features. To avoid over-fitting issues in classification procedure, the train/test performances of LDA classifiers were computed by iteratively removing features based on their statistical effect sizes (t-values) in the SWLDA. The results of this stage of feature selection are shown in Figure 8(a). Based on the train/test performance curve, a final set of 17 EEG features including 13 spectral powers and 4 coherences was selected for the workload classification (Figure $8(\mathrm{~b}))$. The individualized LDA coefficients were trained on the data of the baseline experiment based on the 17 selected features which are listed in Table 3 for the workload prediction of this experiment.

The first row of Figure 9 represents the results of workload estimation for one of the subjects (S3) in the three admittance setups with high-, low-, and variable-damping. According to the spatial characteristics of the predicted workload classes, three different zones including gross, fine, and the transition zone were specified for statistical analysis as depicted in Figure 9(c). For each subject, the predicted LDA scores are normalized into z-scores and then averaged within each defined zone for the three damping conditions to be used for the statistical analysis. Figures 9(d) and (e) illustrate the mean predicted workload scores for the different damping conditions and task zones across the subjects, respectively.

To examine the main effects of different damping conditions and task zones on the EEG-based predicted workload, a $3 \times 3$ [Damping (High, Low, and Variable) $\times$ Zone (Gross, Transition, and 
Table 3. List of Selected Features

\begin{tabular}{|c|c|c|c|}
\hline Type & Band & Channel & $\mid$ t-statistics $\mid$ \\
\hline \multirow{13}{*}{ Spectral Power Density } & Theta & $\mathrm{Cz}$ & 2.98 \\
\hline & \multirow{2}{*}{ Low-Alpha } & P3 & 4.55 \\
\hline & & C3 & 2.67 \\
\hline & \multirow{2}{*}{ High-Alpha } & F3 & 4.49 \\
\hline & & $\mathrm{C} 3$ & 6.53 \\
\hline & \multirow{3}{*}{ Low-Beta } & $\mathrm{T} 4$ & 2.90 \\
\hline & & F4 & 2.45 \\
\hline & & P3 & 2.50 \\
\hline & \multirow{3}{*}{ High-Beta } & T4 & 3.18 \\
\hline & & T3 & 1.35 \\
\hline & & $\mathrm{F} 4$ & 2.72 \\
\hline & \multirow{2}{*}{ Gamma } & $\mathrm{C} 3$ & 3.21 \\
\hline & & $\mathrm{P} 4$ & 3.44 \\
\hline \multirow{4}{*}{ Spectral Coherence } & High-Alpha & $\mathrm{Fz}-\mathrm{Pz}$ & 3.31 \\
\hline & High-Beta & Fz-T3 & 7.01 \\
\hline & \multirow{2}{*}{ Gamma } & Fz-T3 & 3.02 \\
\hline & & Fz-O1 & 2.93 \\
\hline
\end{tabular}
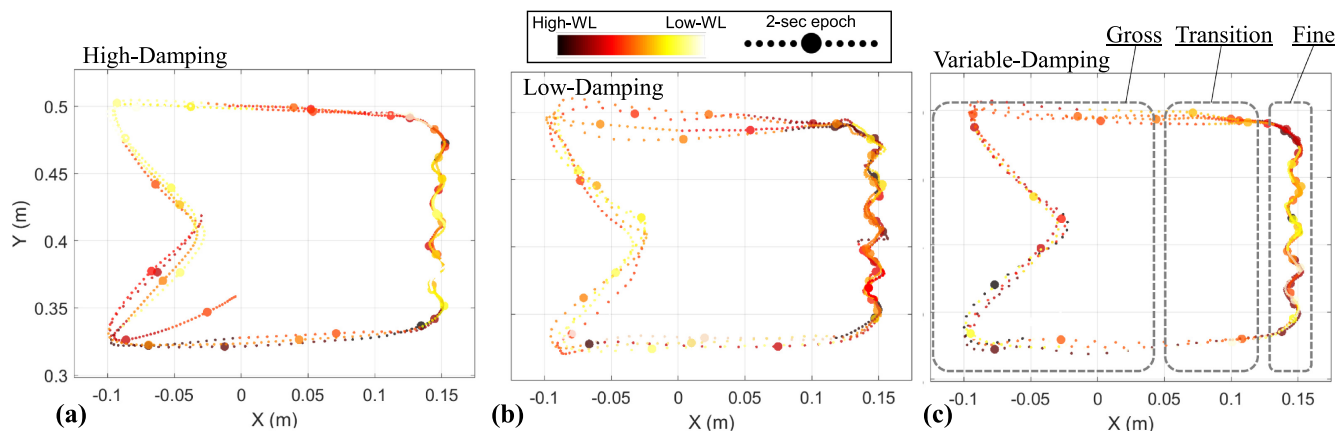

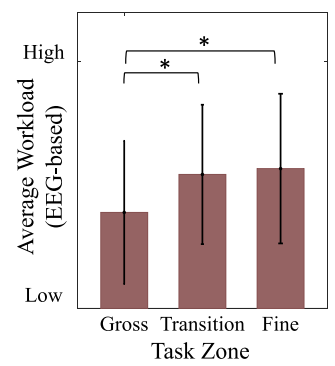

(d)

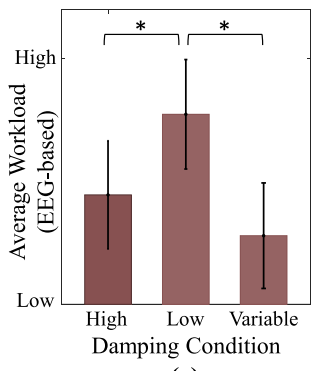

(e)

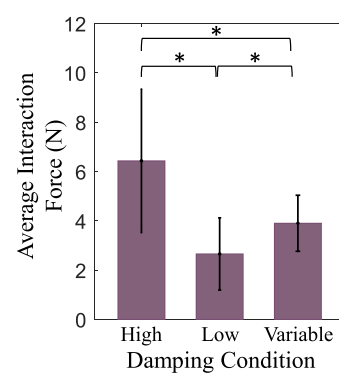

(f)

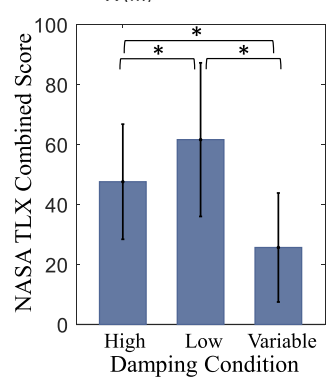

(g)

Fig. 9. The results of EEG-based workload analysis for the secondary experiment. The first row shows the results of LDA scores for a representative subject (S3) with (a) high-damping, (b) low-damping, and (c) variabledamping. The bar plots of second row indicate the average results over the 10 subjects. (d) The effect of Task Zone on the predicted workload score. $(\mathrm{e}-\mathrm{g})$ Comparison of the effect of Damping condition on predicted workload, interaction force, and NASA-TLX (subjective workload). 
Fine)] repeated-measures ANOVA was performed. As the follow-up tests, multiple pairwise comparisons were conducted for a better interpretation of the significant main effects. Furthermore, Bonferroni adjustment of confidence intervals was used for pairwise comparisons to protect the analysis from Type-I error when conducting multiple tests.

The results revealed the significant main effects of both Damping $(F(2,8)=54.4, p<.001)$ and Zone $(F(2,8)=7.2, p=.018)$ on the workload with a non-significant Damping $\times$ Zone interaction. According to the results of the baseline experiment, we expected to observe a lower EEG-based workload in the case of variable-damping. Pairwise comparisons revealed that the workload in the variable-damping was only lower than the low-damping $(p<.001)$ as depicted in Figure 9(d). However, no statistically significant difference between the variable-damping and high-damping was observed. This might be due to the fact that the time spent in the fine zone was much more than the gross zone, particularly for the variable-damping, which had low-damping in the gross and high-damping in the fine zone (the average co-manipulation duration of the gross and fine zones were $6.7 \pm 1.6$ and $20.5 \pm 5.1$ seconds, respectively). This can be realized from the data point concentration in Figure 9(c) as well. As a result, it can be hypothesized that the short duration of the gross zone did not allow the subjects to completely adapt to the new dynamics with a low damping before going back to the fine zone with a high damping. The overall results suggest that if a constant damping is required, high-damping setups are more suitable than a low-damping, particularly if the task involves fine co-manipulations.

To further explore the effect of damping on the user, repeated-measures ANOVAs were applied on the average interaction forces and the overall workload score obtained from NASA TLX questionnaire separately. Bonferroni correction was used to protect the analysis from Type-I error caused by multiple comparisons. The results are shown in the bar plots of Figure 9(f). As expected, the statistical results revealed a significant main effect of damping on the interaction forces $(F(2,8)=77.4, p<.001)$. Pairwise comparisons indicated that the mean interaction force in the high-damping was higher than the variable-damping $(p<.001)$; and in the variable-damping, it was higher than the low-damping $(p<.001)$. These results indicate that although for the EEGbased workload prediction no significant difference between the high- and variable-damping was observed, average interaction forces in the variable-damping were improved significantly. This was consistent with the perceived task-load of the subjects obtained from NASA TLX, which is depicted in Figure 9(g).

Interestingly, in terms of the task zones, an increased workload was observed in the transition between the gross and fine zone as shown in Figure 9(d). In fact, the mean workload in the transition and fine zone were higher than the gross zone $(p<.01$ and $p<.05$, respectively). This is mainly due to the increased attention that was required when switching between the gross and fine co-manipulation and accordingly large changes in the motion velocity. This suggests that in the design of variable admittance controllers, careful considerations should be made to avoid increased workload when type of manipulation is subjected to changes and motion over-shooting is likely to occur due to the sudden changes of velocity.

\section{DISCUSSION}

The behavioral and subjective assessment revealed that an appropriate admittance setup (virtual damping) is task-dependent. Although decreasing virtual damping resulted in lower interaction forces in both fine and gross co-manipulation, a higher damping in the fine co-manipulation revealed superior performances. In terms of the relationship between workload and performance, typically excessive increase in the task difficulty/workload leads to marked degradation in the task performance making the performance as an indirect estimation of workload. This was consistent with our subjective assessment that revealed a significant interaction between the two 
experimental factors (Manipulation-Type and Virtual-Damping). According to the results of the behavioral and subjective assessment, a binary classification problem was defined. Low-damping gross manipulation and high-damping fine manipulation were considered as low-workload condition (class-1), and the two other tasks were labeled as high-workload condition (class-2). This resulted in a task-independent binary classification in terms of manipulation type, since each of the classes contained both co-manipulation types.

In the context of workload estimation (especially cognitive-workload), many studies have examined the relationship between task-load and EEG spectral power measures. In the studies that involved visual search, working memory, focused attention, or multi-tasking, the most prominent influence of an increased workload was observed in prefrontal theta and parietal alpha power (see Borghini et al. [2014]). However, only left-parietal (P3) alpha power appeared in the selected feature set of this study. This is mainly due to the different attentional and executive resources that are required for visuomotor tasks [Popovic et al. 2015]. As a result, this study mostly involved cerebral cortical activations specific to sensory-motor rather than cognitive demands, as identified with C3 higher-alpha power in Figure 8(b).

The stepwise linear discriminant analysis ended up with a set of 17 features mostly in the highfrequency bands (beta and gamma). Cortical activity in gamma and higher-beta band are associated with localized sensory integration and correlate with sensory processing demand [Von Stein and Sarnthein 2000]. Right temporal, parietal, and occipital activity in these bands are indicative of visuospatial processing, and they usually increase with motor task difficulty [Goodale and Milner 1992]. Interestingly, Rietschel et al. observed a similar activation and connectivity patterns in a study that cognitive-motor task difficulty was manipulated in a Tetris game [Rietschel et al. 2012]. Given high degree of sensory integration during co-manipulation tasks, particularly visuospatial processing, beta and gamma activity as a function of motor control difficulty seems reasonable.

Among the selected features, Fz coherence with left-temporal (T3) region has a strong relationship with "psychomotor efficiency" hypothesis in psycho-physiology. This hypothesis states that the skilled performance of experts, particularly in sports, is the outcome of efficient networking between brain regions [Deeny et al. 2009; Hatfield et al. 2004]. In fact, non-essential inter-regional interactions between the motor planning and other regions of the brain are required to be suppressed for a higher performance. Among these inter-regional interactions, Fz-T3 communication is strongly associated with verbal-analytical processes (instructional self-talk) and is essential for early stages of motor learning, whereas it will be attenuated when the movements become highly learned. Most likely these indices can be an indication of difficulty in a physical human-robot interaction when it is not intuitive/well-designed.

In terms of the classification, the obtained classification accuracy was relatively high (with linear classifiers an average of $>81 \%$ ) and significantly higher than chance $(50 \%)$. This indicates that EEG monitoring can be a promising technique to assess users' state during physical interactions. There are at least two factors contributing to the observed variations in the classification rates between subjects: (i) Individual differences in the level of expertise; not all the data epochs of an experimental condition represent the overall workload level. (ii) Individual differences in brain anatomy resulting in electrode misalignment and functional differences in how participants perform the task.

The secondary experiment was conducted by combining fine and gross manipulation tasks to demonstrate how the classification results of the baseline experiment can be used to explore co-manipulation tasks with variable admittance/impedance control strategies. Although a simple rule was used to form the variable-damping strategy with a smooth switching between low- and high-damping, the lower average workload in the variable-damping demonstrated its effectiveness. In case of constant damping, the high-damping approach was shown to be more suitable 
than low-damping if the co-manipulation involves fine positioning. Moreover, temporal workload information captured by the EEG classifier could provide us with the possibility of workload analysis in different parts of the task. For instance, an increased workload was observed at the transition from gross to fine manipulation as well as altering controller dynamics. Indeed, such temporal information of the users' states can help designers to improve the quality of physical human-robot interactions.

Although continuous EEG signals are highly sensitive to the user's mental state and suffer from a low signal-to-noise ratio, our results indicate that the proposed model is a viable source of information regarding operator workload for improving the neuroergonomic aspects of pHRI. In general, the continuous classification of operator workload can be employed in two different directions: either as an offline workload analysis tool or as a real-time adaptation feedback. Two potential areas that can benefit from an offline workload analysis are the assessment of different variable impedance/admittance control methods across different tasks, and the personalization of pHRI (e.g., subject-specific admittance control [Gopinathan et al. 2017]).

Moreover, an EEG-based workload prediction obtained from real-time data can serve as an additional modality in pHRI. For example, robot assistance of motor learning/recovery can be accelerated by dynamic difficulty adjustment to improve user engagement and performance. However, only relying on an EEG-based classification as the main modality to dynamically change admittance/impedance parameters might not be easily achievable given the low signal-to-noise ratio of EEG signals. For instance, in Aricò et al. [2016], a moving average of 30 seconds was applied on the workload indices to trigger assistance in an air-traffic control scenario; or in Walter et al. [2017], the task difficulty was adjusted only between the trials of an arithmetic-problemsolving experiment.

\section{CONCLUSION}

In this article, we presented an experimental study to investigate the validity of EEG-based workload classification as a continuous measure for physical human-robot cooperation. For this purpose, the effect of controller dynamics on EEG signals and perceived workload were examined in a co-manipulation task with admittance control. Two types of manipulation consisting of gross and fine movements with low and high virtual damping were performed by the subjects. Performance and subjective measures revealed that a suitable damping level depends on the type of manipulation. Although interaction forces decreased by lower virtual damping in the fine co-manipulation, task performance and accordingly perceived workload degraded with low damping.

The experimental design of this study resulted in a task-independent binary classification problem in the sense that both low- and high-workload conditions contained the fine and gross comanipulation. The relationships of the selected features with visuospatial processing and psychomotor efficiency support the validity of the feature selection procedure. The high accuracy rate of classification results for each individual indicates the reliability of EEG signals to assess user's state during physical human-robot cooperation.

Furthermore, we conducted a secondary experiment by combining fine and gross manipulation and performed EEG-based workload analysis for constant and variable damping controllers. We demonstrated how the obtained classifiers from the baseline experiment can be used as a continuous objective measure for workload analysis in more general scenarios. We showed that a variable controller can enhance operator workload; however, careful considerations should be made when transitions between different dynamics are required.

The results of this study would enable designers to assess the effectiveness of different variable compliance methods in terms of perceived interaction workload by the users. Moreover, real-time implementation of these classifiers can be used as an input to adaptive interaction techniques 
for decision making. For example, motor-learning rate can be enhanced by using real-time motor workload classifiers for adapting system behavior in learning of motor skills.

In the presented study, we only investigated the interaction forces in terms of the average magnitude of forces over each task. However, the quality of physical interaction can be further investigated by analyzing different smoothness measures in the time and frequency domain. For example, instead of the binary classification of workload a continuous force/motion smoothness measure can be considered as an indirect measure of motor control difficulty for regression analysis [Memar and Esfahani 2018]. Studying the learning effect and human adaptation to different comanipulation dynamics are other possible future directions that can be investigated and compared with EEG patterns. Moreover, the current study has focused on co-manipulation tasks involving limited degrees of freedom (DoF). In the future, we aim to compare different adaptation techniques by using EEG-based workload analysis in 6-DoF human-robot cooperation. Moreover, the generalizability of the obtained results in other human-robot cooperation applications can be studied in the future.

Furthermore, robot-assisted motor learning is another research domain that the results of this study can utilize to assess interaction designs. Recently, several studies aimed to use EEG signals to adapt robot assistance in rehabilitation such that an optimal level of workload can be maintained and motor-learning rate maximized [Badesa et al. 2012; Esfahani et al. 2015; George et al. 2012; Guerrero et al. 2013; Novak et al. 2014]. For instance, George et al. [2012] used haptic assistance in a path-following task based on an EEG workload index. Although the workload index of their study identified the mental arithmetic load rather than motor control difficulty, an overall improved performance was obtained for eight subjects by combining haptics and EEG monitoring.

\section{REFERENCES}

Kazunori Akizuki and Yukari Ohashi. 2015. Measurement of functional task difficulty during motor learning: What level of difficulty corresponds to the optimal challenge point? Hum. Move. Sci. 43 (Oct. 2015), 107-117. DOI: https://doi.org/ 10.1016/j.humov.2015.07.007

A. M. Amjad, D. M. Halliday, J. R. Rosenberg, and B. A. Conway. 1997. An extended difference of coherence test for comparing and combining several independent coherence estimates: theory and application to the study of motor units and physiological tremor. F. Neurosci. Meth. 73, 1 (1997), 69-79.

Charles W. Anderson, Erik A. Stolz, and Sanyogita Shamsunder. 1998. Multivariate autoregressive models for classification of spontaneous electroencephalographic signals during mental tasks. IEEE Trans. Biomed. Eng. 45, 3 (1998), $277-286$.

Pietro Aricò, Gianluca Borghini, Gianluca Di Flumeri, Alfredo Colosimo, Stefano Bonelli, Alessia Golfetti, Simone Pozzi, Jean-Paul Imbert, Géraud Granger, Raïlane Benhacene et al. 2016. Adaptive automation triggered by EEG-based mental workload index: A passive brain-computer interface application in realistic air traffic control environment. Front. Hum. Neurosci. 10 (2016), 539.

Francisco Javier Badesa, Ricardo Morales, Nicolás Garcia-Aracil, José María Sabater, Carlos Perez-Vidal, and Eduardo Fernandez. 2012. Multimodal interfaces to improve therapeutic outcomes in robot-assisted rehabilitation. IEEE Trans. Syst., Man, Cyber., Part C (Appl. Rev.) 42, 6 (2012), 1152-1158.

Ali Bashashati, Mehrdad Fatourechi, Rabab K. Ward, and Gary E. Birch. 2007. A survey of signal processing algorithms in brain-computer interfaces based on electrical brain signals. F. Neural Eng. 4, 2 (2007), R32.

Nezha Bennis and Agnès Roby-Brami. 2002. Coupling between reaching movement direction and hand orientation for grasping. Brain Res. 952, 2 (2002), 257-267. DOI : https://doi.org/10.1016/S0006-8993(02)03250-X

Chris Berka, Daniel J. Levendowski, Michelle N. Lumicao, Alan Yau, Gene Davis, Vladimir T. Zivkovic, Richard E. Olmstead, Patrice D. Tremoulet, and Patrick L. Craven. 2007. EEG correlates of task engagement and mental workload in vigilance, learning, and memory tasks. Aviat., Space, Environ. Med. 78, 5 (2007), B231-B244.

C. L. Bethel, K. Salomon, R. R. Murphy, and J. L. Burke. 2007. Survey of psychophysiology measurements applied to humanrobot interaction. In Proceedings of the 16th IEEE International Symposium on Robot and Human Interactive Communication. 732-737.

D. H. Blackwood and W. J. Muir. 1990. Cognitive brain potentials and their application. The British fournal of Psychiatry 157 (1990), 96-101. 
Gianluca Borghini, Laura Astolfi, Giovanni Vecchiato, Donatella Mattia, and Fabio Babiloni. 2014. Measuring neurophysiological signals in aircraft pilots and car drivers for the assessment of mental workload, fatigue, and drowsiness. Neurosci. Biobehav. Rev. 44 (2014), 58-75.

Mike X. Cohen. 2014. Analyzing Neural Time Series Data: Theory and Practice. The MIT Press.

Sean P. Deeny, Amy J. Haufler, Mark Saffer, and Bradley D. Hatfield. 2009. Electroencephalographic coherence during visuomotor performance: A comparison of cortico-cortical communication in experts and novices. F. Motor Behav. 41, 2 (2009), 106-116.

Frédéric Dehais, Emrah Akin Sisbot, Rachid Alami, and Mickaël Causse. 2011. Physiological and subjective evaluation of a human-robot object hand-over task. Appl. Ergon. 42, 6 (2011), 785-791.

Arnaud Delorme and Scott Makeig. 2004. EEGLAB: an open source toolbox for analysis of single-trial EEG dynamics including independent component analysis. J. Neurosci. Meth. 134, 1 (2004), 9-21.

Fotios Dimeas and Nikos Aspragathos. 2015. Reinforcement learning of variable admittance control for human-robot comanipulation. In Proceedings of the IEEE/RSF International Conference on Intelligent Robots and Systems (IROS'15). IEEE, 1011-1016.

Vincent Duchaine and Clement M. Gosselin. 2007. General model of human-robot cooperation using a novel velocity based variable impedance control. In Proceedings of the Second foint EuroHaptics Conference and Symposium on Haptic Interfaces for Virtual Environment and Teleoperator Systems (World Haptics'07). IEEE, 446-451.

Mustafa Suphi Erden and Bobby Marić. 2011. Assisting manual welding with robot. Robot. Comput.-Integr. Manufact. 27, 4 (2011), 818-828.

Ehsan T. Esfahani, Shrey Pareek, Pramod Chembrammel, Mostafa Ghobadi, and Thenkurussi Kesavadas. 2015. Adaptation of rehabilitation system based on user's mental engagement. In Proceedings of the 17th International Conference on Advanced Vehicle Technologies; 12th International Conference on Design Education; 8th Frontiers in Biomedical Devices Conference. ASME. DOI : https://doi.org/10.1115/DETC2015-47720

Ehsan T. Esfahani and V. Sundararajan. 2011. Using brain-computer interfaces to detect human satisfaction in humanrobot interaction. Int. F. Human. Robot. 08, 01 (2011), 87-101.

Ehsan T. Esfahani and V. Sundararajan. 2012. Classification of primitive shapes using brain-computer interfaces. Comput.Aided Des. 44, 10 (2012), 1011-1019.

Fanny Ficuciello, Luigi Villani, and Bruno Siciliano. 2015. Variable impedance control of redundant manipulators for intuitive human-robot physical interaction. IEEE Trans. Robot. 31, 4 (2015), 850-863.

Theo Gasser, Petra Bächer, and Joachim Möcks. 1982. Transformations towards the normal distribution of broad band spectral parameters of the EEG. Electroenceph. Clinic. Neurophys. 53, 1 (1982), 119-124.

Laurent George, Maud Marchal, Loeiz Glondu, and Anatole Lécuyer. 2012. Combining brain-computer interfaces and haptics: Detecting mental workload to adapt haptic assistance. Haptics: Perception, Devices, Mobility, and Communication. Springer, 124-135.

Melvyn A. Goodale and A. David Milner. 1992. Separate visual pathways for perception and action. Trends Neurosci. 15, 1 (1992), 20-25.

Sugeeth Gopinathan, Sonja Kristine Ötting, and Jochen J. Steil. 2017. A user study on personalized stiffness control and task specificity in physical human-robot interaction. Front. Robot. AI 4 (2017), 58.

S. Grafakos, F. Dimeas, and N. Aspragathos. 2016. Variable admittance control in pHRI using EMG-based arm muscles co-activation. In Proceedings of the IEEE International Conference on Systems, Man, and Cybernetics (SMC'16). 001900001905.

Carlos Rodriguez Guerrero, Juan Carlos Fraile Marinero, Javier Perez Turiel, and Victor Muñoz. 2013. Using human state aware robots to enhance physical human-robot interaction in a cooperative scenario. Comput. Meth. Prog. Biomed.112, 2 (2013), 250-259.

Sandra G. Hart and Lowell E. Staveland. 1988. Development of NASA-TLX (Task Load Index): Results of empirical and theoretical research. Adv. Psychol. 52 (1988), 139-183.

Bradley D. Hatfield, Amy J. Haufler, Tsung-Min Hung, and Thomas W. Spalding. 2004. Electroencephalographic studies of skilled psychomotor performance. F. Clinic. Neurophys. 21, 3 (2004), 144-156.

Bo Hjorth. 1970. EEG analysis based on time domain properties. Electroenceph. Clinic. Neurophys. 29,3 (1970), 306-310.

N. Hogan. 1984. Impedance control: An approach to manipulation. In Proceedings of the American Control Conference. 304313.

Ryojun Ikeura, Hiroshi Monden, and Hikaru Inooka. 1994. Cooperative motion control of a robot and a human. In Proceedings of the 3rd IEEE International Workshop on Robot and Human Communication (RO-MAN'94). IEEE, 112-117.

Robert Jenke, Angelika Peer, and Martin Buss. 2014. Feature extraction and selection for emotion recognition from EEG. IEEE Trans. Affect. Comput. 5, 3 (2014), 327-339.

Yufeng Ke, Hongzhi Qi, Feng He, Shuang Liu, Xin Zhao, Peng Zhou, Lixin Zhang, and Dong Ming. 2014. An EEG-based mental workload estimator trained on working memory task can work well under simulated multi-attribute task. Front Hum. Neurosci. 8 (2014). 
Dean J. Krusienski, Eric W. Sellers, François Cabestaing, Sabri Bayoudh, Dennis J. McFarland, Theresa M. Vaughan, and Jonathan R. Wolpaw. 2006. A comparison of classification techniques for the P300 Speller. f. Neural Eng. 3, 4 (2006), 299.

Dana Kulic and Elizabeth A. Croft. 2007. Affective state estimation for human-robot interaction. IEEE Transa. Robot. 23, 5 (2007), 991-1000.

Alexandre Lecours, Boris Mayer-St-Onge, and Clément Gosselin. 2012. Variable admittance control of a four-degreeof-freedom intelligent assist device. In Proceedings of the IEEE International Conference on Robotics and Automation (ICRA'12). IEEE, 3903-3908.

Timothy D. Lee, Stephan P. Swinnen, and Deborah J. Serrien. 1994. Cognitive effort and motor learning. Quest 46, 3 (1994), 328-344. DOI : https://doi.org/10.1080/00336297.1994.10484130

D. P. Losey and M. K. O’Malley. 2018. Trajectory deformations from physical human-robot interaction. IEEE Trans. Robot. 34, 1 (Feb. 2018), 126-138.

Panadda Marayong and Allison M. Okamura. 2004. Speed-accuracy characteristics of human-machine cooperative manipulation using virtual fixtures with variable admittance. Human Factors 46, 3 (2004), 518-532.

Geoffrey McLachlan. 2004. Discriminant Analysis and Statistical Pattern Recognition. Vol. 544. John Wiley \& Sons.

A. H. Memar and E. T. Esfahani. 2018. EEG correlates of motor control difficulty in physical human-robot interaction: A frequency domain analysis. InProceedings of the IEEE Haptics Symposium (HAPTICS'18). 229-234. DOI : https://doi.org/ 10.1109/HAPTICS.2018.8357181

Alexander Mortl, Martin Lawitzky, Ayse Kucukyilmaz, Metin Sezgin, Cagatay Basdogan, and Sandra Hirche. 2012. The role of roles: Physical cooperation between humans and robots. Int. F. Robot. Res. 31, 13 (2012), 1656-1674. DOI : https:// doi.org/10.1177/0278364912455366

Antonio Moualeu and Jun Ueda. 2015. A model for operator endpoint stiffness prediction during physical human-robot interaction. f. Hum.-Robot Interact. 4, 3 (Dec. 2015), 170-193. DOI : https://doi.org/10.5898/JHRI.4.3.Moualeu

Domen Novak, Benjamin Beyeler, Ximena Omlin, and Robert Riener. 2014. Workload estimation in physical human-robot interaction using physiological measurements. Interact. Comput. 27, 6 (2014), 616-629.

Djordje Popovic, Maja Stikic, Theodore Rosenthal, David Klyde, and Thomas Schnell. 2015. Sensitive, diagnostic, and multifaceted mental workload classifier (PHYSIOPRINT). In Proceedings of the International Conference on Augmented Cognition. Springer, 101-111.

Lei Qin and Bin He. 2005. A wavelet-based time-frequency analysis approach for classification of motor imagery for braincomputer interface applications. F. Neural Eng. 2, 4 (2005), 65. Retrieved from http://stacks.iop.org/1741-2552/2/i=4/a= 001.

Jeremy C. Rietschel, Matthew W. Miller, Rodolphe J. Gentili, Ronald N. Goodman, Craig G. McDonald, and Bradley D. Hatfield. 2012. Cerebral-cortical networking and activation increase as a function of cognitive-motor task difficulty. Bio. Psycholo. 90, 2 (2012), 127-133.

Rong Song, Kai-yu Tong, Xiaoling Hu, and Wei Zhou. 2013. Myoelectrically controlled wrist robot for stroke rehabilitation. f. NeuroEng. Rehab. 10, 1 (10 June 2013), 52. DOI : https://doi.org/10.1186/1743-0003-10-52

Ramesh Srinivasan, William R. Winter, Jian Ding, and Paul L. Nunez. 2007. EEG and MEG coherence: Measures of functional connectivity at distinct spatial scales of neocortical dynamics. F. Neurosci. Meth. 166, 1 (2007), 41-52.

Emanuel Todorov. 2004. Optimality principles in sensorimotor control. Nat. Neurosci. 7, 9 (2004), 907-915.

Antony Tran, Dikai Liu, Ravindra Ranasinghe, Marc Carmichael, and Chuanbo Liu. 2015. Analysis of human grip strength in physical human robot interaction. Procedia Manufact. 3 (2015), 1442-1449. DOI : https://doi.org/10.1016/j.promfg.2015. 07.320

Jan B. F. van Erp, Hans (J. A.) Veltman, and Marc Grootjen. 2010. Brain-based Indices for User System Symbiosis. Springer London, 201-219. DOI : https://doi.org/10.1007/978-1-84996-272-8_12

Astrid Von Stein and Johannes Sarnthein. 2000. Different frequencies for different scales of cortical integration: From local gamma to long range alpha/theta synchronization. Int. f. Psychophys. 38, 3 (2000), 301-313.

Carina Walter, Wolfgang Rosenstiel, Martin Bogdan, Peter Gerjets, and Martin Spüler. 2017. Online EEG-based workload adaptation of an arithmetic learning environment. Front. Hum. Neurosci. 11 (2017), 286.

Received November 2017; revised August 2019; accepted October 2019 